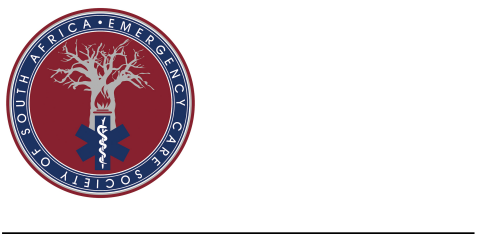

ARTICLE INFORMATION

Received: 24 January 2020

Revision received: 11 April 2020

Accepted: 28 April 2020

Keywords:

Emergency Medical Services

triage

dispatch

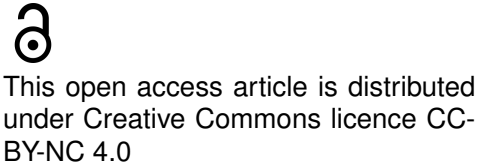

BY-NC 4.0

ISSN:2709-3786

\section{Pre-hospital over-triage and potential contributing factors in Cape Town, South Africa}

\author{
David John McAlpine ${ }^{1,}{ }^{*}$, Peter Hodkinson ${ }^{1}$, Julian Fleming ${ }^{2}$ \\ ${ }^{1}$ Division of Emergency Medicine, Faculty of Health Sciences, Uni- \\ versity of Cape Town \\ ${ }^{2}$ Western Cape Government Department of Health
}

"Corresponding author: davidjohnmcalpine@gmail.com, Old Main Building, Groote Schuur Hospital, Main Road Observatory, 7925

\section{ABSTRACT}

Background: Inappropriate dispatch of urgent ambulances by callcentre personnel causes an unnecessary drain on existing resources. How often these urgent dispatches are appropriate has not been evaluated in the lower-middle-income setting, nor have factors been assessed that contribute to these decisions. This study aimed to establish the rates of pre-hospital over-triage in Cape Town, South Africa, and to assess the call-centre decision-making processes.

Methods: This was a descriptive, retrospective study examining all urgent ambulance dispatches made from a large public sector ambulance call centre in Cape Town over a single month. This urgent dispatch was then compared to the on-scene South African Triage Scale (SATS) score assigned by the pre-hospital personnel to assess which cases were 'over-triaged' by the call taker. Factors potentially contributing to the call taker's decision were also analysed and included the time of day, nature of presenting complaint, and the call taker's training and experience - all of which may have affected the rates of over-triage.

Results: In the course of one month in 2017, 4,169 urgent calls were assessed; of these, 2,701 were over-triaged $(58.48 \%)$. The over-triage rate was similar during the day $(58.02 \%)$ and night $(59.11 \%)$. The most regularly over-triaged complaint was obstetric and gynaecological $(84.87 \%)$, followed by motor vehicle accidents $(65.70 \%)$; the lowest rate was for cardiac call-outs (47.12\%). We reviewed the 38 highest workload call takers, and found no statistically significant factors that contributed to higher levels of over-triage when reviewing higher levels of training (Ambulance Emergency Assistant $62.16 \%$, no medical training $59.42 \% ; \mathrm{p}=0.669)$, more years as a call taker $(<2$ years $59.32 \%$, $>5$ years $60.23 \% ; \mathrm{p}=0.932)$, and more years working in the field $(0$ years $59.36 \%,>5$ years $63.66 \%$; $\mathrm{p}=0.305$ ).

Conclusion: The rates of pre-hospital over-triage in Cape Town are marginally lower than those described internationally. The nature of the complaint had a strong impact on these rates, notably in terms of trauma and gynaecological disorders. The call taker's training and years of experience did not have a statistically significant impact on decision-making.

McAlpine DJ, Hodkinson P, Fleming J. Pre-hospital over-triage and potential contributing factors in Cape Town, South Africa. South African Journal of Pre-hospital Emergency Care. 2020; 1(2):6-12. doi:10.24213/1-2-3876 


\section{BACKGROUND}

South Africa's healthcare system is under pressure from the triple burden of high levels of communicable and non-communicable diseases, and interpersonal violence. ${ }^{1,2}$ As a result, it is essential for emergency services to achieve a balance between servicing those most in need of urgent medical care and providing the best healthcare to the greatest number of people. ${ }^{3}$ Many unwell South Africans rely on Emergency Medical Services (EMS) as their primary method of accessing health services, placing EMS at the forefront of equitable access to healthcare. It therefore becomes important to examine whether this service is being optimally utilised and consider which factors that might affect its efficiency. South Africa has both a private and public healthcare system that functions largely independently of one another. For the purpose of this study, only the public pre-hospital system was considered.

The system in Cape Town works as follows: when an emergency call for an ambulance is made, a call taker based in an Emergency Call Centre (ECC) makes a rapid decision on the urgency of the ambulance dispatch. The most urgent calls are allocated by the call taker as 'Priority 1' (P1), and an ambulance is dispatched with lights and sirens with the aim of arriving at the patient within 15 minutes. 'Priority 2' (P2) is allocated for less urgent cases with the aim of arrival within $60 \mathrm{~min}-$ utes. Extensive resources are required to enable this prompt P1 response time, particularly since meeting the 15-minute target is used as the primary performance indicator for ambulance efficacy in the province. ${ }^{4}$ It is therefore important to examine whether the decision to dispatch a P1 ambulance is subsequently proven to be correct. Are a large number of urgent ambulances incorrectly dispatched to relatively stable patients - known as 'over-triage' - at the expense of the service as a whole?

The majority of international studies conducted on this phenomenon thus far have reported prehospital over-triage rates of between $64 \%$ and $84.6 \% .{ }^{5-10}$ While the American College of Surgeons has stipulated acceptable rates of over-triage in trauma patients as $25-35 \%$, no similar guidelines exist for non-trauma patients. $5,11,12$ Hence, it remains uncertain what rates of over-triage are acceptable. Few studies to date have considered this phenomenon in low-and-middle-income countries (LMICs) such as South Africa. The majority of information on over-triage comes from wellresourced settings where the call takers make use of computerised triage tools, following a protocolised set of questions based on the patient's present- ing complaint. ${ }^{5,13-15}$ The two most commonly used are the Medical Priority Dispatch System (MPDS) and the Criteria Based Dispatch (CBD) system. ${ }^{13}$ Many studies have thus focused on the efficacy of these protocolised triage tools rather than on the call takers themselves, who hold greater importance in the LMIC setting where triage tools are not routinely used.

In Cape Town, the majority of call takers are not trained medical personnel, and they do not have a protocolised triage tool to follow. Instead, they use a combination of elements from the South African Triage Scale (SATS), rough guidelines, prior experience, and in-service training. ${ }^{16,17}$ As such, it becomes important to look not only at whether they are making the correct decisions but also at factors that may lead to more effective dispatching.

This study aimed to assess the levels of over-triage in Cape Town and identify whether presenting complaints, time of day, and the level of the call taker's training and experience had an effect on over-triage rates.

\section{DESIGN AND METHODS}

\section{Study Design}

The study design was a retrospective, descriptive study of P1 ambulance dispatches over a single month.

\section{Study Population and Sample}

The study reviewed a convenience sample of all calls made in a calendar month to the Cape Town ECC. This centre fields all calls for the public sector EMS service in the city. Information was obtained from a digital call-centre database regarding both the number and nature of call-outs, as well as the triage colour assigned on scene. A secondary outcome looked at the call takers themselves who were on duty during the study period and who had dispatched 20 or more P1 ambulances during the month. This information was obtained from the same database.

\section{Methods}

P1 dispatches were compared to SATS triage colours allocated on scene to assess the accuracy of the call-out. The SATS looks at vital signs, presenting complaint and mechanism of injury, and allocates a colour based on the urgency of the case, with red being the most urgent, then orange, yellow and finally green as the most non-urgent. As there is no exact equivalency between the call centre's triage of P1/P2 and the SATS triage colour used by the ambulance crew on scene, this study 
deemed all those P1 cases subsequently triaged as both 'green' and 'yellow' as being 'non-urgent' and therefore 'over-triaged' by the call taker. This decision was based on the fact that, in hospital, patients with SATS triage colour 'red' should be seen immediately and 'orange' should be seen within ten minutes of arriving; a P1 ambulance aims to arrive within 15 minutes after being dispatched. ${ }^{18}$ 'Yellow' patients should be seen within an hour and 'green' patients within four hours; P2 ambulances aim to arrive within 60 minutes after being dispatched. Hence, although imperfect, a relative equivalency can be seen between these two sepa- rate triage systems.

As a secondary outcome, the study looked for other factors that might affect allocation accuracy, such as time of day and the call taker's experience. The time of day was recorded as either 'night shift' (19h00 to 0700) or 'day shift' (0700 to 19h00), which reflect the 12-hour shifts worked by both call centre and ambulance personnel. The nature of the presenting complaints was grouped from the EMS database classifications into larger categories, chosen at the investigators' discretion (Table 1) and the rates of over-triage for each of these groups were assessed.

Table 1: Groupings of EMS Classifications

\begin{tabular}{|c|c|}
\hline Triage Category & EMS Classification \\
\hline Trauma (Other) & $\begin{array}{l}\text { Accidental injury, assault, burns and corrosives, self- } \\
\text { harm,weapon }\end{array}$ \\
\hline Trauma (MVA/PVA) & $\begin{array}{l}\text { Bus/taxi, cyclist, motorcyclist, pedestrian, train and railway } \\
\text { accident, truck/heavy vehicle }\end{array}$ \\
\hline Abdominal & Abdominal complaint, dehydration, diarrhoea, and vomiting \\
\hline Cardiac & Cardiac chest pain, cardiac complaint, CPR in progress \\
\hline Respiratory & Respiratory complaint, unable to breathe \\
\hline Obstetric \& Gynaecological & Gynaecological problem, obstetric problem \\
\hline Neurological (Convulsions) & Convulsions \\
\hline Neurological (Other) & Confusion, neurological complaint \\
\hline Unresponsive & Unresponsive \\
\hline Other & $\begin{array}{l}\text { Fever, envenomation, wilderness search,allergy, non- } \\
\text { traumatic bleeding, self-harm poisoning, dermatological, } \\
\text { non-cardiac pain, industrial, informal structures, residential, } \\
\text { musculoskeletal pain }\end{array}$ \\
\hline
\end{tabular}

Information was also collected via a voluntary survey about the call-taking personnel themselves and included their levels of medical training: whether they had none at all (simply six weeks of in-service training as a call taker), had passed a four-week Basic Ambulance Assistant (BAA) course, or whether they had a six-month Ambulance Emergency Assistant (AEA) qualification. Their years of experience working as EMS crew and years working in the call centre were also examined, together with the available data to assess if any particular set of call-taker characteristics lead to lower rates of over-triage.

This research was conducted with ethical approval from the University of Cape Town Human Research Ethics Committee (HREC 849/2017), as well as approval from the Western Cape Department of Health Emergency Medical Services.

\section{Data Analysis}

Both descriptive and inferential statistics were used, employing Microsoft Office Excel 2015 Version 15.12.3 (Microsoft Corporation, Redmond, Washington) and Dell Statistica 2015 Version 13.0 (Dell, Round Rock, Texas). Analysis of variance parametric testing was applied to assess the statistical significance between means where a normal distribution was present.

\section{RESULTS}

The study investigated a total of 44,139 calls made within Cape Town during August 2017, since August represents an 'ordinary' month, free from the confounders of school holidays and tourism spikes. Once the relevant exclusion criteria were applied (Figure 1) the final sample size was 4,619 cases. Of these, 2,701 P1 cases were coded on scene as 'green' or 'yellow' for an overall call taker overtriage rate of $58.48 \%(\mathrm{p}=0.068)$. The number of cor- 
rectly triaged cases (red or orange) was 1,918 for a rate of $41.52 \%$. More P1 dispatches took place during the day shift at 2,506 (54.25\%) versus 2,113
(45.75\%) at night, but similar rates of over-triage were seen in both of these groups with day shift rates at $58.02 \%$ and night shifts at 59.11

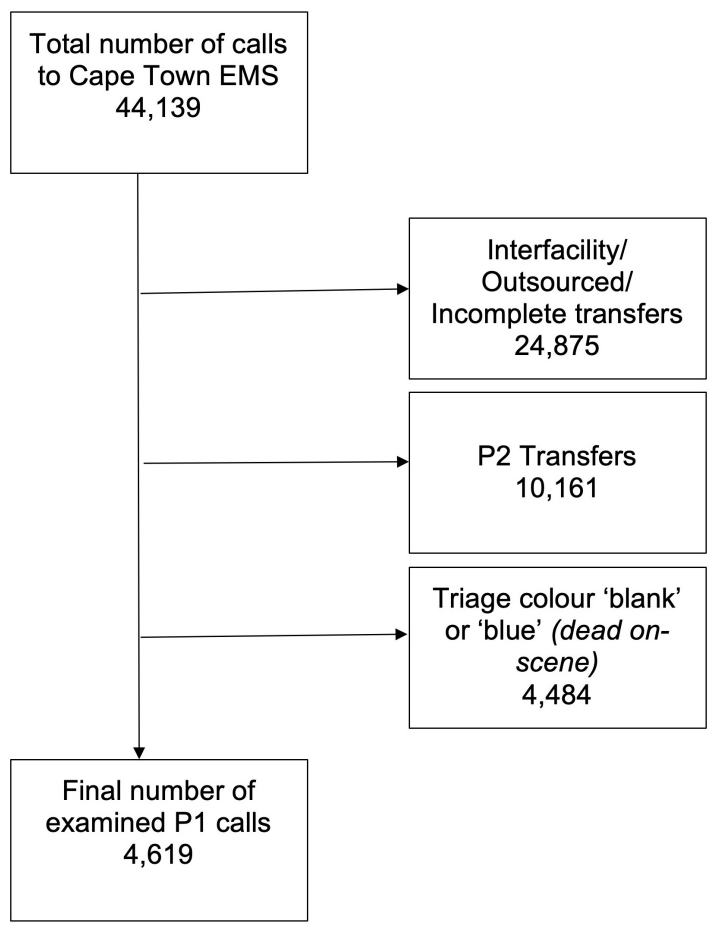

Figure 1: Data Inclusion/Exclusion Flow Diagram

Differences in rates of over-triage were evi- the next most frequently over-triaged scenarios, dent based on the patient's presenting complaint. which were motorvehicle accidents (MVAs) and The highest rates of over-triage were seen in pedestrian-vehicle accidents (PVAs) at $65.7 \%$. The those with obstetric and gynaecological com- lowest rates of over-triage were observed in those plaints, at $84.87 \%$. This was notably higher than with cardiac complaints at $47.12 \%$ (Table 2 ).

Table 2: Percentages of Over- and Correct Triage by Presenting Complaint and Total Cases

\begin{tabular}{llll}
\hline Presentation & Total Cases & Over-triaged \% & \% Total P1s \\
\hline O\&G Complaint & 235 & 84.9 & 5.2 \\
Trauma (MVA/PVA) & 544 & 65.7 & 11.9 \\
Abdominal Complaint & 62 & 63.5 & 1.4 \\
Other & 213 & 61.3 & 4.7 \\
Trauma: Other & 782 & 59.7 & 17.2 \\
Respiratory Complaint & 1281 & 58.2 & 28.1 \\
Unresponsive & 277 & 55.2 & 6.1 \\
Confusion, Neurological Complaint & 435 & 49.7 & 9.6 \\
Convulsions & 515 & 49.4 & 11.3 \\
Cardiac Complaint & 275 & 47.1 & 6 \\
\hline
\end{tabular}

O\&G = Obstetrics and gynaecology; MVA = motorvehicle accident; PVA = pedestrian-vehicle accident

Of the 96 call takers who dispatched P1 call-outs, 40 had fielded 20 or more calls that met inclusion criteria. Of these 40 , two had resigned, and the investigators were unable to obtain sufficient infor- mation and therefore excluded them for a final total of 38 call takers. Their levels of medical training, years of experience in the call centre and years working in ambulances are illustrated in Table 3. 
Table 3: Call-taker Descriptors: Mean Percentage of Over-triage and Call Taker Numbers

\begin{tabular}{llll}
\hline Qualification & Mean (SD) \% Over-triaged Calls & Call Takers & $\mathbf{p}$ \\
\hline None & $59.4(6.5)$ & 19 & 0.669 \\
BAA & $59.7(5.0)$ & 14 & \\
AEA & $62.2(7.6)$ & 5 & \\
Total & $59.9(6.0)$ & 38 & $\mathbf{p}$ \\
\hline Call-taker Experience (Years) & Mean (SD) \% Over-triaged Calls & Call Takers & 0.932 \\
\hline$<2$ & $59.3(2.6)$ & 5 & \\
$2-5$ & $59.6(5.1)$ & 13 & \\
$>5$ & $60.2(7.3)$ & 20 & $\mathbf{p}$ \\
Total & $59.9(6.0)$ & 38 & 0.305 \\
\hline EMS Experience (Years) & Mean (SD) \% Over-triaged Calls & Call Takers & \\
\hline None & $59.4(5.6)$ & 28 & \\
$<2$ & $56.4(4.5)$ & 3 & \\
$2-5$ & $63.7(7.9)$ & 3 & \\
$>5$ & $63.2(8.6)$ & 38 & \\
Total & $59.9(6.0)$ & & \\
\hline
\end{tabular}

EMS = Emergency medical services; $\mathrm{SD}=$ standard deviation

The combined number of calls fielded by these call takers was 3,853 . There were no statistically significant differences in mean over-triage rates between levels of basic training $(\mathrm{p}=0.669)$, years of call takers' experience $(\mathrm{p}=0.932)$, and years working in the field $(\mathrm{p}=0.305)$.

\section{DISCUSSION}

The study showed that rates of over-triage by call takers in Cape Town were lower than those seen internationally $(64 \%-85 \%) .{ }^{5-10}$ While this is certainly encouraging, it is unclear if this occurred at the expense of high levels of under-triage - where a non-urgent ambulance is sent to a patient in urgent need. Clearly, the lower the threshold for dispatching an urgent ambulance, the higher the rates of over-triage will be, but the lower the rates of under-triage will be. This is the most likely reason for the marginally lower rates compared to higherincome settings - their centres and triage tools are more conservative in sending out unnecessary P1s in order to miss fewer urgent cases. Examining under-triage was beyond the scope of this paper but certainly warrants further research.

In LMICs where resources are limited, this balance between acceptable levels of over- and undertriage is paramount. By establishing current rates and setting goals for future ones, prehospital services can look to optimise these resources. Likewise, the potential to establish new systems for improvement, such as the introduction of a low-cost triage tool for the existing call takers, can be considered.
Gynaecological conditions had the highest rates of over-triage by a considerable margin, likely indicating an ongoing drive to reduce perinatal mortality, as a continually measured yardstick of a region's healthcare system. Vehicle accidents were over-triaged by $65.70 \%$, reflecting the difficulties call takers experienced in assessing the severity of a polytrauma case over the telephone. The least over-triaged presentation was for cardiac complaints - consistent with other studies - and likely reflects the ambulance personnel's reticence to triage a chest pain as 'non-urgent' despite potentially normal vital signs.

It was found in this small sample of call takers that there was no association between the call taker's experience or training and over-triage. A statistically non-significant trend reflected that there were marginally higher rates of over-triage among call takers with greater experience. Further research examining a longer timeline with more eligible call takers and a greater number of calls might bear more significant findings.

\section{LIMITATIONS}

The study had several limitations. The first of these relates to the lack of equivalency between the triage systems used by the EMS call takers and those used by the health providers on scene. While it makes intuitive sense that a P1 call should be viewed in the same 'urgent' category as those triaged 'red' and 'orange', the fact that the study is comparing two distinct prioritisation systems is potentially problematic, as is their incongruence with international systems. SATS has not been 
validated for pre-hospital use, so its effectiveness in this setting has not yet been established. ${ }^{17,19,20}$ The study also did not review whether the healthpersonnel on scene were correctly applying the SATS nor the accuracy of their categorisation; incorrect assessments on scene might skew the data towards higher levels of over-triage.

This study was purely a quantitative examination of a potentially qualitative process. Examining the calls themselves would have been useful in ascertaining the reasons behind call taker's decisions in over-triaging certain cases. The extent to which language barriers and cultural differences affect individual calls has been established in recent papers, but its effect on the accuracy of pre-hospital triage remains uncertain. ${ }^{21,22}$ Likewise, it is unclear whether some cases of over-triage are due to the person making the call exaggerating the clinical picture in order to get a faster ambulance. Further qualitative research in this field would shed light on this issue.

Presenting complaints were logged on the EMS datasheet into smaller coherent groups. Certain presentations cannot easily be attributed to a single system (for example 'unresponsive' and 'confusion' can both be due to pathology in any number of organ systems); similarly, certain categories within the EMS database were difficult to place at all, such as 'industrial', 'self-harm' and 'noncardiac pain'. Moreover, age was not documented in the EMS datasheet, so how this may affect the call taker's decision is not understood. In particular, it is uncertain if calls involving children received higher prioritisation than those involving adults. Improved data capturing is required to better examine this important issue.

Finally, the study did not look at the equally important levels of under-triage to assess if nonurgent ambulances are being dispatched and causing delayed access to healthcare for acutely ill individuals. This balance between low levels of both over- and under-triage is the crux of pre-hospital dispatch and certainly warrants further research.

\section{CONCLUSION}

The rates of over-triage in Cape Town would seem to be lower than those found in better-resourced settings. While this is certainly encouraging, it was beyond the scope of this study to assess if this was at the expense of high levels of under-triage. Likewise, the absence of international guidelines or data from other LMICs makes it uncertain if these are, independently, acceptable or not. As such, there should be a continual drive to improve call takers' ability to make accurate decisions. Al- though this study was not able to determine which characteristics result in the most effective call taker, this is also an easy target for potential future improvement. Wide variations in rates of over-triage based on the primary complaint is certainly an area that could be targeted and investigated for further improvement.

\section{ACKNOWLEDGMENT}

The authors would like to acknowledge Colleen Saunders for her extensive help with the statistics in this paper.

\section{CONFLICTS OF INTEREST}

The authors report no conflicts of interest.

\section{AUTHOR CONTRIBUTIONS}

Conceptualisation of research: PH, DM; data collection: DM, JF; data analysis: DM; drafting and revision of manuscript: DM, PH, JF; final approval of the manuscript: DM, PH, JF.

\section{REFERENCES}

1. Mayosi BM, Benatar SR. Health and health care in South Africa-20 years after Mandela. N Engl J Med. 2014;371:1344-53.

2. South Africa: WHO statistical profile. Geneva: World Health Organisation, 2018. (https : / / www . who . int / gho / countries / zaf / country_profiles / en/) (visited on 04/06/2018).

3. South Africa. Constitution of the Republic of South Africa Act No 108 of 1966

4. Annual Report 2019. Cape Town: Western Cape Department of Health, 2019. (https : / / www . westerncape.gov . za / dept / health / documents / annual_reports / 2019) (visited on 03/05/2020).

5. Dami F, Golay C, Pasquier M, V.Fuchs, Carron PN, Hugli O. Prehospital triage accuracy in a criteria based dispatch centre. BMC Emerg Med. 2015;15, 32.

6. Ek B, Edström P, Toutin A, Svedlund M. Reliability of a Swedish pre-hospital dispatch system in prioritizing patients. Int Emerg Nurs. 2013;21:143-9.

7. Hoikka M, Länkimäki S, Silfvast T, AlaKokko TI. Medical priority dispatch codescomparison with National Early Warning 
Score. Scand J Trauma Resusc Emerg Med. 2016;24, 142.

8. Khorram-Manesh A, Montán KL, Hedelin A, Kihlgren M, Örtenwall P. Prehospital triage, discrepancy in priority-setting between emergency medical dispatch centre and ambulance crews. Eur J Trauma Emerg Surg. 2011;37:73-8.

9. Lu TC, Chen YT, Ko PC, et al. The demand for prehospital advanced life support and the appropriateness of dispatch in taipei. Resuscitation 2006;71:171-9.

10. Sporer KA, Youngblood GM, Rodriguez RM. The ability of emergency medical dispatch codes of medical complaints to predict als prehospital interventions. Prehosp Emerg Care 2007;11:192-8.

11. Resources for optimal care of the injured patient. Chicago: Committee on Trauma, American College of Surgeons, 2014. (https : / / www . facs . org / / media / files / quality 20 programs / trauma / vrc 20 resources/resources $\% 20$ for \% 20 optimal $\div 20$ care. ashx) (visited on 03/11/2018).

12. Mann NC, Schmidt TA, Cone DC. Defining research criteria to characterize medical necessity in emergency medical services: a consensus among experts at the neely conference. Prehosp Emerg Care 2004;8:138-53.

13. Deakin CD, Sherwood DM, Smith A, Cassidy M. Does telephone triage of emergency (999) calls using Advanced Medical Priority Dispatch (AMPDS) with Department of Health (DH) call prioritisation effectively identify patients with an acute coronary syndrome? An audit of 42,657 emergency calls to Hampshire Ambulance Service NHS Trust. Emerg Med J. 2006;23:232-5.

14. Hardeland C, Olasveengen TM, Lawrence R, et al. Comparison of Medical Priority Dispatch (MPD) and Criteria Based Dispatch
(CBD) relating to cardiac arrest calls. Resuscitation 2014;85:612-6.

15. Culley LL, Henwood DK, Clark JJ, Eisenberg MS, Horton C. Increasing the efficiency of emergency medical services by using criteria based dispatch. Ann Emerg Med. 1994;24:867-72.

16. Twomey M, Wallis LA, Thompson ML, Myers JE. The South African Triage Scale (adult version) provides reliable acuity ratings. Afr J Emerg Med. 2012;2:3-12.

17. Twomey M, Cheema B, Buys $\mathrm{H}$, et al. Vital signs for children at triage: a multicentre validation of the revised South African Triage Scale (SATS) for children. S Afr Med J. 2013;103:304-8.

18. The South African Triage Scale (SATS) training manual 2012. Cape Town: Western Cape Department of Health, 2012. (https : / / emssa.org.za/wp-content/uploads / 2011 / 04 / SATS - Manual - A5 - LR spreads . pdf) (visited on 04/20/2018).

19. Rosedale K, Smith ZA, Davies H, Wood D. The effectiveness of the South African Triage Score (SATS) in a rural emergency department. S Afr Med J. 2011;101:537-40.

20. Wallis PA, Wood SBGD, Bruijns S, Vries S de, Balfour C, Cape Triage Group. The Cape Triage Score - a triage system for South Africa. S Afr Med J. 2006;96:53-6.

21. Anest T, Stewart de Ramirez S, Balhara KS, Hodkinson P, Wallis L, Hansoti B. Defining and improving the role of emergency medical services in Cape Town, South Africa. Emerg Med J. 2016;33:557-61.

22. Kironji AG, Hodkinson $P$, Stewart de Ramirez $\mathrm{S}$, et al. Identifying barriers for out of hospital emergency care in low and low-middle income countries: A systematic review. BMC Health Serv Res. 2018;18, 291. 